J. Phys. IV France 134 (2006) 629-634

(C) EDP Sciences, Les Ulis

DOI: 10.1051/jp4:2006134097

\title{
An experimental method of measuring the confined compression strength of high-performance concretes to analyse their ballistic behaviour
}

\author{
P. Forquin ${ }^{1}$, A. Árias ${ }^{2}$ and R. Zaera ${ }^{2}$ \\ ${ }^{1}$ Laboratory of Physics and Mechanics of Materials, University Paul-Verlaine of Metz, \\ Ile du Saulcy, 57045 Metz Cedex 1, France \\ 2 Department of Continuum Mechanics and Structural Analysis, University Carlos III \\ of Madrid, 28911 Leganés, Madrid, Spain
}

\begin{abstract}
The test known as "quasi-oedometric compression" consists of the compression of a cylindrical specimen confined in a thick vessel. In this work, an original methodology is proposed to deduce the radial stress and strain within the specimen using hoop strains measured on the external surface of the vessel, taking into account its elasto-plastic deformation. On one hand the spherical and deviatoric behaviours of two concretes are deduced. On the other hand, their ballistic behaviour is analysed using impact tests. These experiments are simulated numerically by the plasticity model of Krieg, Swenson and Taylor, and the features of the model are identified by the previous confined compression tests. The capacity of the model to describe the ballistic behaviour of such materials is shown in a comparison of the numerical simulations with the ballistic tests.
\end{abstract}

\section{INTRODUCTION}

A good knowledge of the behaviour of geomaterials under confined pressure is a prerequisite for any analysis and modelling of their ballistic properties. In the impact of a projectile on a massive target, a field of confined compression is created ahead of the projectile. The strength of the material under high pressure and the law of compaction (irreversible diminution of the volume) determine the depth of penetration of the projectile into the target [1,2], and this is why confined compression tests have been developed.

Compression tests known as "quasi-oedometric" were developed on geomaterials (their name refers to the very weak radial displacement during the test). A cylindrical concrete specimen is placed in a confinement vessel. Subjected to axial compression, the specimen expands radialy and exerts pressure on the confinement vessel. The test produces a greater axial stress and a greater radial stress on the specimen, so the hydrostatic confinement pressure varies considerably during the test and the resistance to these different confinement pressures can be measured. Several experimental devices for quasioedometric compression were set up [3, 4]. Burlion [3] performed those tests assuming an elastic deformation of the vessel so the radial strain and stress of the specimen were deduced from the microdeformation of the vessel (a few hundredths \%) measured by gauges on its outer surface. A quasioedometric compression device was also developed with smaller vessels [4]. This device was adapted to Hopkinson Bars to measure the dynamic strength of the MB50 microconcrete. The analysis in axial stress showed a very limited influence of the rate of loading on the stress level, even at a strain rate that reached $400 \mathrm{~s}^{-1}$. In this paper, a quasi-oedometric device and a new methodology of analysis are presented and used with two concretes. After that, their ballistic performances are discussed in relation with their properties measured under confined compression. Finally a model is used to simulate numerically the ballistic tests, and the results in terms of depth of penetration are compared with the experimental results. 


\section{THE QUASI-OEDOMETRIC DEVICE}

Fig. 1 shows the set-up of the quasi-oedometric compression tests practised in this study. To eliminate any void inside the vessel, a CHRYSOR® C6120 epoxy resin was extruded along with the insertion of the specimen. The specimen enclosed in the confinement vessel is subjected to axial loading. The pressure cell of a Servosis ${ }^{\mathrm{TM}}$ hydraulic universal testing machine relays the axial load to the specimen, and from this the axial stress can be deduced. An analogic extensometer is placed between the two compression cylinders. With this set-up we obtain the axial strain of the specimen after subtracting the displacement caused by the elastic deformation of the compression cylinders. Four gauges are placed on the confinement vessel (fig. 1b).
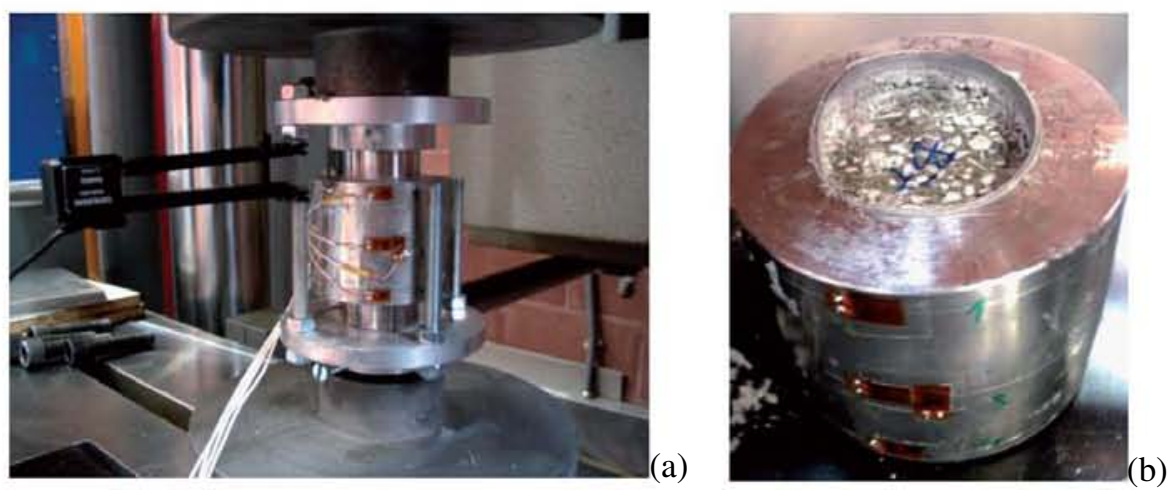

Figure 1. The quasi-oedometric compression device (a) and the gauges placed on the confinement vessel (b).

\section{A NEW METHOD OF ANALYSIS OF QUASI-GEDOMETRIC COMPRESSION TESTS}

To evaluate the evolution of the strength of concrete one must determine the lateral pressure exerted by the specimen against the inner wall of the vessel. The relation between the radial stress imposed by the specimen on the internal wall of the vessel, and the hoop strain of the external wall, is deduced from two calculations made with Abaqus/Implicit. The behaviour law used to model the steel of the vessel is determined by tensile tests. In these calculations, the pressure is applied along a height $h_{\text {press }}$ $\left(h_{\text {press }}=34\right.$ or $40 \mathrm{~mm}$ ). From these calculations, the evolution of the radial stress is obtained as a function of the external hoop strain at the symmetry plane $(\mathrm{z}=0)$ through two functions $f_{40}$ and $f_{34}$, each one calibrated for each load case:

$$
\bar{\sigma}_{\text {radial }}\left(h_{\text {press }}=40\right)=f_{40}\left(\varepsilon_{\theta \theta}^{(z=0, e x t)}\right) \quad \text { and } \quad \bar{\sigma}_{\text {radial }}\left(h_{\text {press }}=34\right)=f_{34}\left(\varepsilon_{\theta \theta}^{(z=0, \text { ext })}\right)
$$

The height $h_{\text {press }}$ takes into account the shrinkage of the specimen caused by the compaction of the concrete during the test. In addition, this shrinkage (the average axial strain of the specimen) is measured directly. From these two equations we deduce the internal radial stress as a function of the axial strain, using the following linear ratio:

$$
\bar{\sigma}_{\text {radial }}\left(\bar{\varepsilon}_{\text {axial }}, \varepsilon_{\theta \theta}^{(z=0, e x t)}\right)=\left(1-\frac{\bar{\varepsilon}_{\text {axial }}}{\varepsilon_{\text {ref }}}\right) f_{40}\left(\varepsilon_{\theta \theta}^{(z=0, e x t)}\right)+\left(\frac{\bar{\varepsilon}_{\text {axial }}}{\varepsilon_{\text {ref }}}\right) f_{34}\left(\varepsilon_{\theta \theta}^{(z=0, e x t)}\right)
$$

in which $\bar{\varepsilon}_{\text {axial }}$ is the nominal axial strain, $\varepsilon_{r e f}$ the reference strain $\left(\varepsilon_{r e f}=-0.15\right)$, and $f_{40}\left(\varepsilon_{\theta \theta}^{z=0, e x t}\right)$, $f_{34}\left(\varepsilon_{\theta \theta}^{z=0, e x t}\right)$ are the functions of equations (1) and (2). In the same way, the expression for the average 
internal radial strain of the specimen in the vessel is deduced as follows:

$$
\bar{\varepsilon}_{\text {radial }}=\frac{2}{3}\left(1-\bar{\varepsilon}_{\text {axial }}\left(1+\frac{\bar{\varepsilon}_{\text {axial }}}{2}\right)\right) \alpha_{0}^{0} \varepsilon_{\theta \theta}^{(z=0, \text { ext })}+\frac{\left(1+\bar{\varepsilon}_{\text {axial }}\right)^{2}}{3} \alpha_{20}^{18} \varepsilon_{\theta \theta}^{(z=18, \text { ext })}
$$

The two strains $\left(\varepsilon_{\theta \theta}^{(z=0, e x t)}, \varepsilon_{\theta \theta}^{(z=18, e x t)}\right)$ are those measured in the tests by the strain gauges attached to the vessel and $\alpha_{0}^{0}$ and $\alpha_{20}^{18}$ are coefficients which are on an average 2.745 and 2.20 respectively for the vessels used. Since the internal radial strain and stress in the concrete are known, one can deduce the deviatoric stress $\left(\bar{\sigma}_{\text {deviatoric }}\right)$ and the hydrostatic pressure:

$$
\bar{\sigma}_{\text {deviatoric }}=\left|\bar{\sigma}_{\text {axial }}-\bar{\sigma}_{\text {radial }}\left(\bar{\varepsilon}_{\text {axial }}, \varepsilon_{\theta \theta}^{\text {ext }}\right)\right| \quad \text { and } \quad \bar{P}_{\text {hydrostatic }}=-\frac{1}{3}\left(\bar{\sigma}_{\text {axial }}+2 \bar{\sigma}_{\text {radial }}\left(\bar{\varepsilon}_{\text {axial }}, \varepsilon_{\theta \theta}^{\text {ext }}\right)\right)
$$

and the deviatoric and volumetric strains are given by the formulae:

$$
\bar{\varepsilon}_{\text {deviatoric }}=\frac{2}{3}\left|\ln \left(1+\bar{\varepsilon}_{\text {axial }}\right)-\bar{\varepsilon}_{\text {radial }}\right| \quad \text { and } \quad \bar{\varepsilon}_{\text {volumetric }}=\ln \left(1+\bar{\varepsilon}_{\text {axial }}\right)+2 \bar{\varepsilon}_{\text {radial }}
$$

Thus, with only the data of the axial force, the axial strain, and 2 external hoop strains measured by three gauges attached to the vessel, we can obtain the deviatoric behaviour (i.e., the evolution of the deviatoric stress) and the spherical behaviour (the variation of the volumetric strain with the hydrostatic pressure). This was validated on one hand by numerical simulations of the quasi-oedometric compression of concrete specimens, particularly with regard to the possible influence of friction between the specimen and the vessel and the influence of the interface product Chrysor®. On the other hand, the experimental set-up was validated by the quasi-oedometric compression tests carried out on a wellknown aluminium alloy. Now it is a question of applying this experimental method to the analysis of the behaviour of two high-performance concretes under confined compression.

\section{RESULTS OF THE QUASI-OEDOMETRIC TESTS PERFORMED WITH 2 CONCRETES}

Since the aim of the study was to determine the ballistic properties of particle-reinforced mortar, two materials were prepared. The first, called M2 is a mortar with no reinforcement, composed of fine sand, cement, silica fumes, water and additive. The mass ratio water/binder (cement + silica fumes) is around 0.41 , and the ratio sand/binder was 2.2. This composition gives a satisfactory relation between malleability and strength. The second type of mortar, the M2M, had the same composition as the first with the addition of alumina ceramic particles $(41.1 \%$ by mass, around $30 \%$ vol.). The cylindrical for simple and quasi-oedometric tests- and cubic -for ballistic tests- samples used in the study were cut and extracted from the interior of two large blocks (around $28 \times 20 \times 6 \mathrm{~cm}^{3}$ ), one from each mortar.

The deviatoric and spherical curves are shown in Fig. 2. A notable rise of strength occurs with the hydrostatic pressure. While the simple compression strength of the M2 mortar is about $67 \mathrm{MPa}$, it rises to over $200 \mathrm{MPa}$ under a hydrostatic pressure of $240 \mathrm{MPa}$ and may even reach $350 \mathrm{MPa}$ under confinement of $460 \mathrm{MPa}$. The rise of strength of the reinforced type M2M is even more spectacular than that of the M2. Under light confinement $\left(P_{\text {hydrostatic }}=50 \mathrm{MPa}\right.$, strength $\left.=100 \mathrm{MPa}\right)$ it is similar, but it is $30 \%$ higher than that of the M2 under strong confinement and reaches $615 \mathrm{MPa}$ under a hydrostatic pressure of $560 \mathrm{MPa}$. So the addition of particles of alumina has a very beneficial effect on the strength of the mortars under confinement. The compaction curves reveal a marked reduction of volume under these high pressures. The volume of the M2 specimens is reduced by about $13 \%$ under a load of $450 \mathrm{MPa}$, while it is below $9 \%$ in the M2M under the same load. Again the presence of the particles is seen to be highly beneficial and it appears to limit the collapse of the pores in the material. 

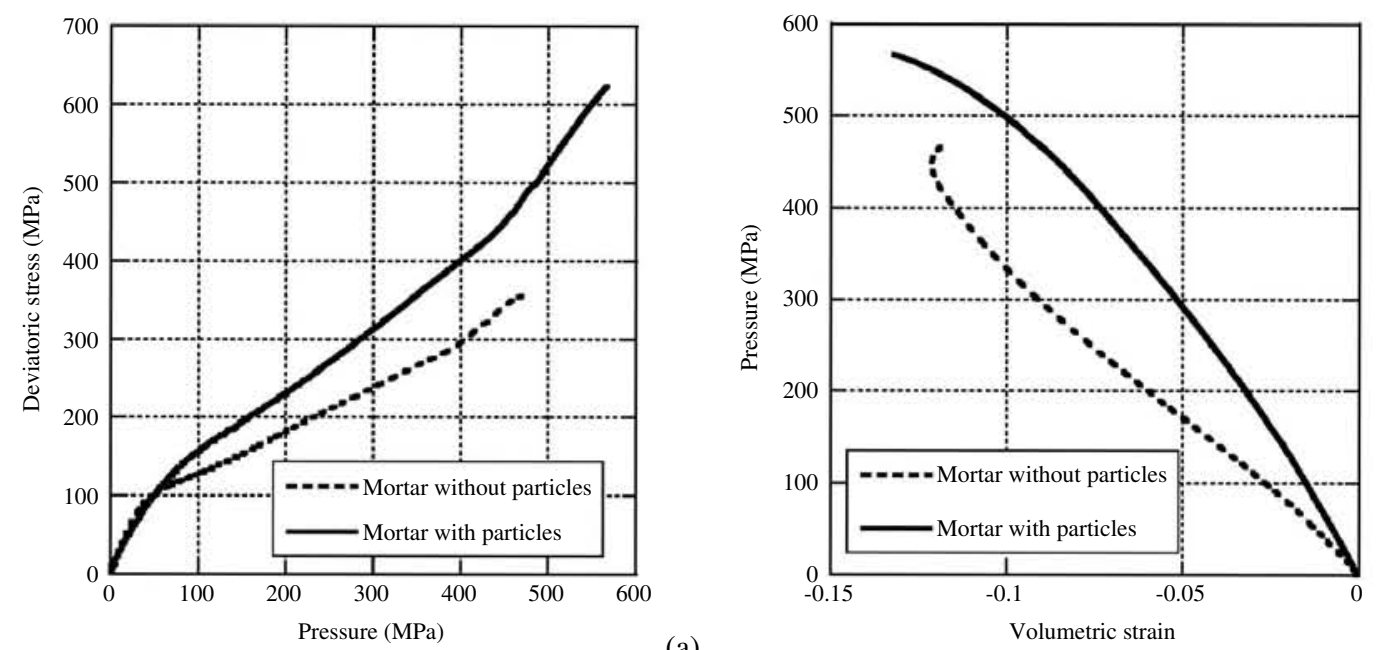

(a)

(b)

Figure 2. Deviatoric behaviour (Fig. 2a) and spherical behaviours (Fig. 2b) of mortars M2 and M2M.

\section{BALLISTIC IMPACT TESTS}

Ballistic impact tests were done with perfectly cylindrical steel projectiles, of diameter $5.3 \mathrm{~mm}$, length $8 \mathrm{~mm}$, and mass $1.2 \mathrm{~g}$, and an exceptionally hard surface (65 HRC). Fired at a speed of $750 \mathrm{~m} / \mathrm{s}$, their velocity was measured with two optic barriers, $2 \mathrm{~m}$ apart. The targets were blocks of $70 \times 70 \times 50 \mathrm{~mm}^{3}$ compatible with the available means of vacuum infiltration and polishing. Each block was placed in an aluminium sarcophagus to restrict movement of the debris of the damaged block after the impact. In addition, to prevent erosion by spalling of the front side of the block, a plywood strip was placed side by side to the face of the block inside the sarcophagus. Previous tests without this strip had shown that it did not affect the depth of penetration. After the impact, each block was infiltrated with a hyperfluid coloured resin. The block was then cut, reinfiltrated and polished for inspection. Two post-mortem analyses are shown in figure 3.

Figure 3 shows the M2 block, impacted at $756 \mathrm{~m} / \mathrm{s}$ and penetrated to a depth of $15.5 \mathrm{~mm}$ as compared to the penetration of $7 \mathrm{~mm}$ of the block with particles, M2M. The path of the first projectile

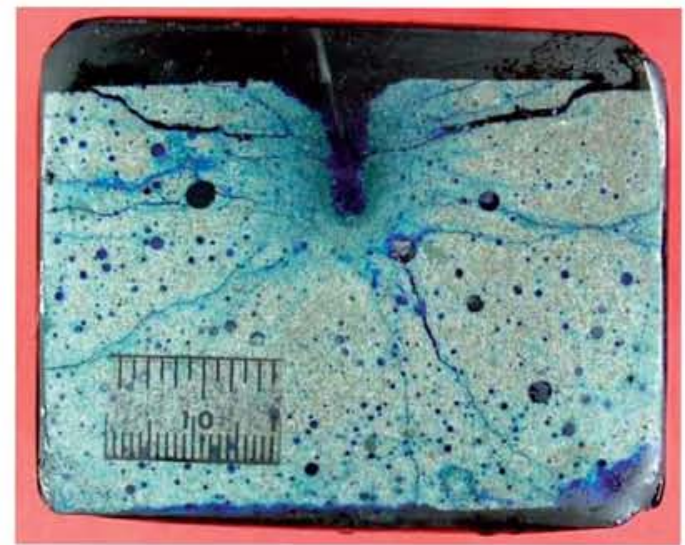

(a) Impact velocity: $756 \mathrm{~m} / \mathrm{s}$, penetration: $15.5 \mathrm{~mm}$

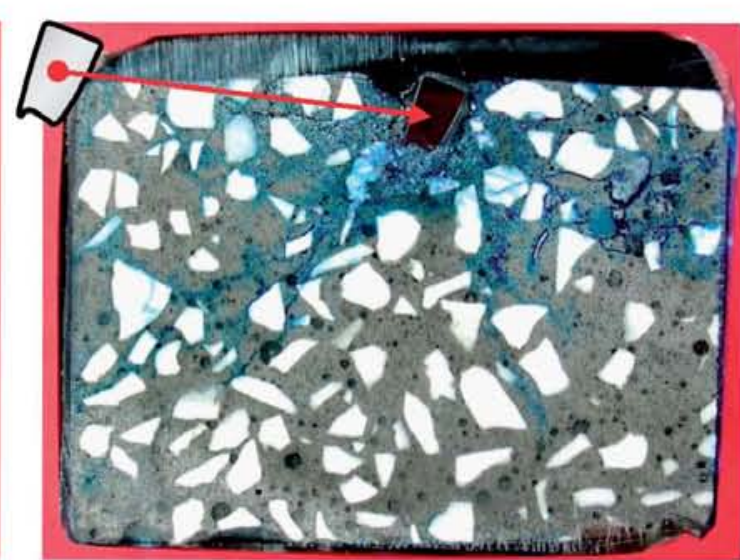

(b) Impact velocity: $749 \mathrm{~m} / \mathrm{s}$, penetration: $7 \mathrm{~mm}$

Figure 3. Ballistic impact of mortars M2 (Fig. 3a) and M2M (Fig. 3b). 
is perfectly straight and perpendicular to the impacted surface (Fig. 3a). The projectile has literally rebounded, although the length of the tunnel is twice that of the projectile. Moreover, the M2 block is heavily damaged by the impact. An intense micro-cracking of the material is visible close to the impact point, transformed into powder in the vicinity of the tunnel. A full fragmentation of the whole block made of numerous long cracks radiating from the tunnel region may also be observed. The set-up in Figure $3 \mathrm{~b}$ (concrete $\mathrm{M} 2 \mathrm{M}$ ) is quite different; the projectile is arrested at less than $7 \mathrm{~mm}$ from the point of impact and no tunnel is visible. This time, the damage close to the front surface is slight even if some fragmentation of alumina particles is visible below the projectile. Furthermore the projectile is slightly inclined. A particle in its pathway has deflected it in spite of its velocity. The surface of the projectile is seen to be clearly deformed by the impact despite its hardness (65 HRC). The depth of penetration is much less than that observed after the impact on an aluminium-alloy armour-plating (5083-H111) by the same projectile at $750 \mathrm{~m} / \mathrm{s}-10.3 \mathrm{~mm}$ - which confirms the excellent ballistic properties of particlereinforced mortars.

\section{NUMERICAL SIMULATION OF THE IMPACT TESTS USING THE “KST” MODEL}

The Krieg, Swenson and Taylor model [5,6] is commonly used to describe the inelastic behaviour of geomaterials. On one hand, the equivalent stress is taken to be a single function of the hydrostatic pressure according to equation 9. On the other hand, a compaction curve is defined by several points $\left(\varepsilon_{v}^{i}, P_{i}\right)$. The hydrostatic pressure is given by interpolation between these points (equation 10):

$$
\sigma_{e q}=\sigma_{y}(P)=\sqrt{a_{0}+a_{1} P+a_{2} P_{2}}
$$

and

$$
P\left(\varepsilon_{v}\right)=P_{i-1} \frac{\varepsilon_{v}-\varepsilon_{v}^{i}}{\varepsilon_{v}^{i-1}-\varepsilon_{v}^{i}}+P_{i} \frac{\varepsilon_{v}-\varepsilon_{v}^{i-1}}{\varepsilon_{v}^{i}-\varepsilon_{v}^{i-1}} \quad \text { for } \varepsilon_{v}^{i-1}>\varepsilon_{v}>\varepsilon_{v}^{i}
$$

These different coefficients $\left(a_{0}, a_{1}, a_{2}, \varepsilon_{v}^{i}, P_{i}\right)$ were identified from the confined compression results (Figs. 3) and are reported in Table 1. An axisymmetric numerical simulation of the ballistic impact tests, with the help of the KST model, is shown in Fig. 4. This simulation uses the finite element code Abaqus/Explicit. By an erosion criteria the elements are eliminated, allowing to reproduce the penetration of the projectile in the target. This method and the mesh were validated through the simulation of the impact of a projectile against a block of aluminium alloy and by comparison with the experimental results. The criterion of erosion considered for the target is that of an equivalent plastic strain of the element equal to $200 \%$. The depth of penetration into the M2 target was of $14.9 \mathrm{~mm}$ (Fig. 4a), which is roughly that observed in the impact tests (Fig. 3a). The penetration of the M2M mortar is slightly overestimated, with a tunnel of $10.2 \mathrm{~mm}$ generated by the simulation whereas the tests gave $7 \mathrm{~mm}$ (Fig. 3b).

\section{CONCLUSION}

An original method is proposed for the analysis of the quasi-oedometric compression tests that takes into account the plastic strain of the vessel and the shortening of the specimen during the compression.

Table 1. Parameters of the Krieg, Swenson and Taylor model.

\begin{tabular}{|l|c|c|}
\hline Parameters & Mortar M2 & Mortar M2M \\
\hline Density, elastic coefficients $(E, v)$ & $3.18 ; 34 \mathrm{GPa} ; 0.2$ & $3.61 ; 48 \mathrm{GPa} ; 0.2$ \\
\hline Deviatoric law $\left(a_{0}, a_{1}, a_{2}\right)$ & $1055 \mathrm{MPa}^{2} ; 165 \mathrm{MPa} ; 0.133$ & $734 \mathrm{MPa}^{2} ; 166 \mathrm{MPa} ; 0.635$ \\
\hline Spherical law $\left(-\varepsilon_{v}^{(1)}, P^{(1)},-\varepsilon_{v}^{(2)}\right.$, & $(0.13 \%, 25 \mathrm{MPa}, 10 \%$, & $(0.24 \%, 60 \mathrm{Mpa}, 8.4 \%$, \\
$\left.P^{(2)},-\varepsilon_{v}^{(3)}, P^{(3)}\right)$ & $314 \mathrm{Mpa}, 12.8 \%, 425 \mathrm{Mpa})$ & $430 \mathrm{Mpa}, 12.8 \%, 545 \mathrm{Mpa})$ \\
\hline
\end{tabular}




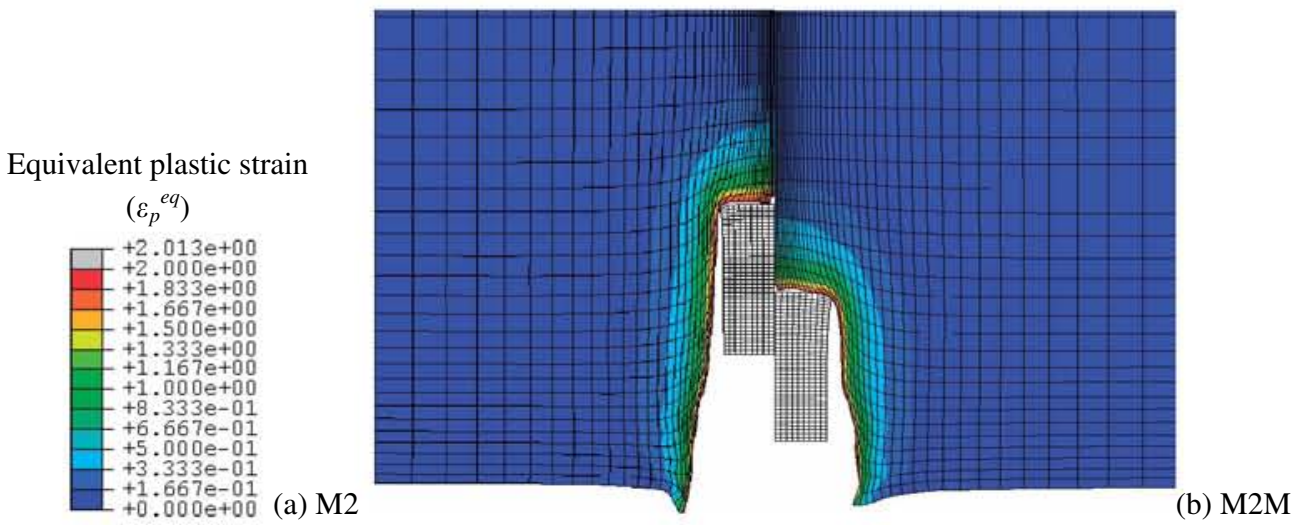

Figure 4. Numerical simulations of the ballistic impact tests using the KST model (impact velocity $=750 \mathrm{~m} / \mathrm{s}$ ).

Quasi-oedometric compression tests were done with two concretes, the second of which was reinforced with angular alumina particles. The tests showed the highly beneficial effect of the presence of the particles, on both the deviatoric strength and the compaction. These results may explain the ballistic properties of the two materials. While the first concrete -without reinforcement- shows only modest ballistic properties and a severe damage under impact, the second type -reinforced by $30 \%$ vol. of particles- reduced considerably the penetration of the projectile without increasing the damage to the target. This correlation between ballistic tests and confined compression tests is explained with the KST plasticity model that describes the deviatoric strength versus pressure and the compaction law of geomaterials under high confinement. The depth of penetration of the unreinforced mortar is correctly predicted whereas the depth of penetration of the reinforced mortar is slightly over-estimated, that may be explained by the presence of ceramic particles with a size close to that of the projectile.

\section{Acknowledgments}

The authors are indebted to the Spanish Comisión Interministerial de Ciencia y Tecnología (Project MAT200203339) for the financial support of this work and to the Délégation Générale pour l'Armement (DGA/France) for the mobility grant provided to P. Forquin.

\section{References}

[1] Hanchak S.J., Forrestal M.J., Young E.R. and Ehrgott J.Q. Perforation of concrete slabs with 48 and $140 \mathrm{MPa}$ unconfined compressive strength. Int. J. Impact Eng., 12(1), (1992) 1-7.

[2] Yankelevsky D.Z. and Dancygier A.N. Uniaxial compressive strength effect on high velocity penetration into thick NSC and HSC targets, symposium ISIEMS (2001).

[3] Burlion N. Compaction des bétons : éléments de modélisation et caractérisation expérimentale. Ph.D. dissertation, Ecole Normale Supérieure de Cachan, Cachan, France, 1997.

[4] Gatuingt F. Prévision de la rupture des ouvrages en béton sollicités en dynamique rapide. Ph.D. dissertation, Ecole Normale Supérieure de Cachan, Cachan, France, 1999.

[5] Krieg R.D. A simple constitutive description for soils and crushable foams. report, SC-DR7260883, Sandia National Laboratory (1978).

[6] Swenson D.V. and Taylor L.M. A finite element model for the analysis of tailored pulse stimulation of boreholes. Int. J. Num. Analyt. Meth. Geomech., 7, (1983) 469-484. 